\title{
Off-Label-Use in der Onkologie - Handlungsalgorithmus bei der Verordnung von Chemotherapie aus Sicht der therapierenden Einrichtung
}

\author{
Galuschka $\mathrm{P}^{1}$, Schoening $\mathrm{T}^{1}$, Thalheimer $\mathrm{M}^{2}$, Seidling $\mathrm{H}^{3}$, Schubert $\mathrm{R}^{4}$, Hoppe-Tichy $\mathrm{T}^{1}$ \\ ${ }^{1}$ Universitätsklinikum Heidelberg, Apotheke \\ ${ }^{2}$ Universitätsklinikum Heidelberg, Qualitätsmanagement und Medizincontrolling \\ ${ }^{3}$ Universitätsklinikum Heidelberg, Klinische Pharmakologie und Pharmakoepidemiologie \\ ${ }^{4}$ Albert-Ludwigs-Universität Freiburg, Lehrstuhl für Pharmazeutische Technologie und Biopharmazie
}

Als Off-Label-Use wird die Anwendung von Arzneimitteln außerhalb der in ihrer Zulassung festgesetzten Rahmenbedingungen bezeichnet [1]. In Deutschland ist im Arzneimittelgesetz $\S 21$ Abs. 1 und $\S 22$ Abs. 1 geregelt, dass Fertigarzneimittel grundsätzlich nur in den Verkehr gebracht werden dürfen, wenn sie eine Zulassung besitzen. Um die Zulassung zu erhalten, muss die Qualität, Wirksamkeit und Unbedenklichkeit des Arzneimittels anhand von Daten aus klinischen Prüfungen nachgewiesen sein ( $\S \S 22,24$ AMG). Durch dieses Verfahren wird die größtmögliche Sicherheit des Patienten angestrebt. Trotzdem kommt es insbesondere in den Bereichen der Pädiatrie, der Onkologie und bei der Behandlung seltener Erkrankungen vor, dass Arzneimittel außerhalb ihrer Zulassung angewendet werden (Off-Label-Use).

Im Bereich der Onkologie wird angenommen, dass im Jahr 2006 bei bis zu 60\% der Patienten in Deutschland ein Arzneimittel off-label verordnet wurde [2]. In etwa $75 \%$ der Fälle stellt jedoch die Indikation den Grund für Off-Label-Verordnungen dar [3].

Laut einer weiteren Studie sind 73\% aller Off-Label-Verschreibungen in den USA ohne Evidenz einer klinischen Effektivität durchgeführt worden, bei weniger als einem Drittel gab es Hinweise auf eine starke wissenschaftliche Evidenz [4]. Hieran wird besonders deutlich, dass die Verordnungen und Gesetze bezüglich Off-LabelUse und Zulassung auch zum Schutz der Patienten vor unkalkulierbaren Risiken entwickelt wurden. Weitere Probleme, die sich für die Krankenhäuser und den verschreibenden Arzt ergeben, sind wirtschaftlicher und haftungsrechtlicher Art [5].

Auch das Leistungs- und Erstattungssystem der gesetzlichen Krankenkassen in Deutschland fußt auf den in der Zulassung festgelegten Therapierichtlinien: Die gesetzliche Krankenversicherung darf ihren Versicherten zur erforderlichen Diagnostik und Therapie grundsätzlich nur Leistungen gewähren, die ausreichend, zweckmäßig und wirtschaftlich sind, sowie das Maß des Notwendigen nicht überschreiten (Wirtschaftlichkeitsgebot im $\S 12$ Abs. 1 SGBV). Daneben ist die Ver- ordnung eines zulassungspflichtigen Arzneimittels zulasten der gesetzlichen Krankenversicherung ausgeschlossen, wenn die Zulassung zum Inverkehrbringen förmlich untersagt wurde, eine $\mathrm{Zu}$ lassungsentscheidung aussteht oder nicht einmal beantragt ist (No-label-use).

\section{Kriterien für die Erstattungsfähig-} keit einer Off-Label-Therapie

In einem ersten wegweisenden Urteil hat bereits 2002 das Bundessozialgericht (BSG) für eine Off-Label-Therapie (B $1 \mathrm{KR}$ 37/00 R vom 19. März 2002) bei folgenden Kriterien die Erstattungsfähigkeit definiert:

- das Vorliegen einer schwerwiegenden, lebensbedrohlichen oder die Lebensqualität auf Dauer nachhaltig beeinträchtigenden Erkrankung,

- das Nichtvorhandensein einer Therapiealternative und

- durch Phase-III-Studien begründete Aussicht auf einen Behandlungserfolg.

Sollten andere Studien zur Wirksamkeit außerhalb der Zulassungsstudien vorliegen, so können auch diese verwendet werden.

Im berühmten „Nikolausurteil“ (AZ 1 BvR 347/98 BVerG vom 6.12.2005) hat das Bundesverfassungsgericht diese Kriterien im Wesentlichen bestätigt, wenn auch teilweise aufgeweicht. So ist die erforderliche Datenlage für einen Off-Label-Use nur noch der „ernsthafte Hinweis“ auf einen „nicht ganz fern liegenden Heilungserfolg“ oder „spürbare positive Einwirkung auf den Krankheitsverlauf “.

\section{Handlungsalgorithmus für die Off-} Label-Prüfung von Arzneimitteln

An dieser Stelle soll ein Handlungsleitfaden für die Off-Label-Prüfung von Arzneimitteln vorgestellt werden, die in der ambulanten onkologischen Therapie von Bedeutung sind. Der Algorithmus soll zudem anerkannten sozialrechtlichen Kriterien standhalten.

Der entwickelte Handlungsalgorithmus (Abb. 1) besteht aus den im Folgenden dargestellten Prüfkriterien (Tab. 1).

\section{Therapiealternativen}

Einer Auswertung der Daten des MDK Westfalen-Lippe zufolge war in den letzten Jahren das Vorhandensein einer Therapiealternative der häufigste Grund für die Ablehnung einer Kostenübernahme bei Off-Label-Use [6]. Daher ist im ersten Schritt zu hinterfragen, ob Therapiealternativen zur Verfügung stehen, die über eine Zulassung bei entsprechender Diagnose verfügen.

\section{Tab. 1 Kriterien, welche bei der Verord-} nungsprüfung berücksichtigt werden sollten.

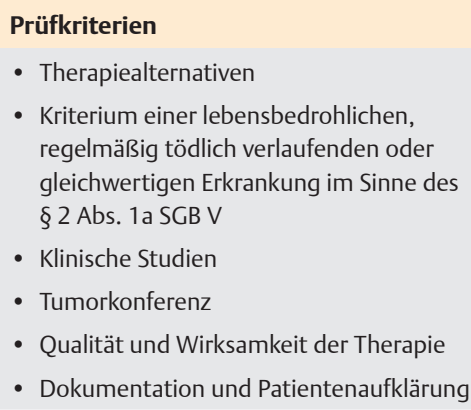


Indikationen der Diagnose und Zulassung stimmen nicht überein

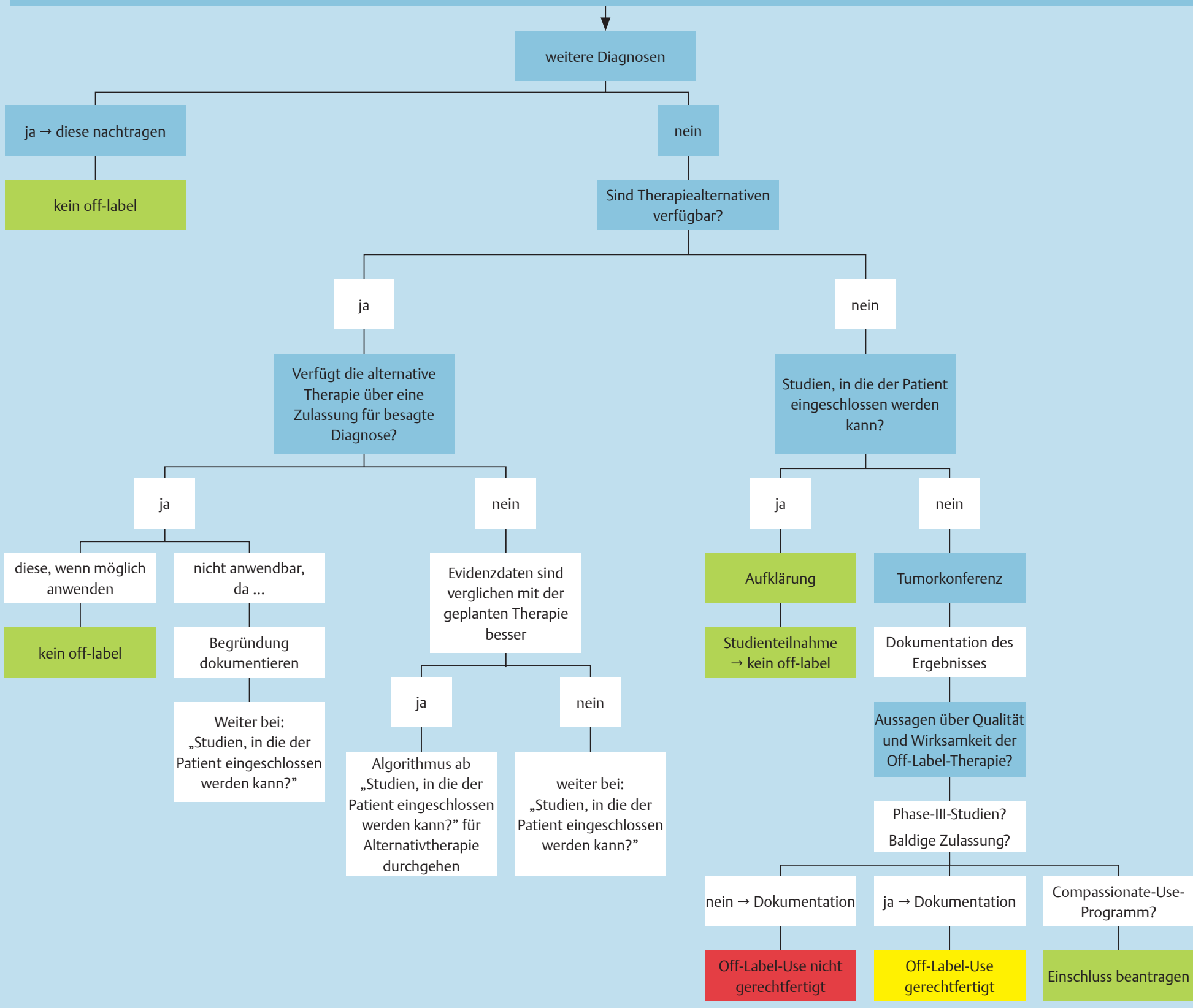

Abb. 1 Algorithmus zur kriterienabhängigen Off-Label-Prüfung.

Stehen keine Therapiealternativen zur Verfügung, so ist nach $\S 2$ Abs. 1a SGB V mit einer Erstattung der Therapiekosten zu rechnen, wenn zusätzlich alle folgenden Kriterien des Algorithmus erfüllt sind.

Kriterium der lebensbedrohlichen, regelmäßig tödlich verlaufenden oder gleichwertigen Erkrankung im Sinne des $\$ 2$ Abs. 1a SGB V

Konnte keine zugelassene Therapiealternative ermittelt werden, muss im Folgenden sichergestellt werden, dass es sich bei der zu therapierenden Erkrankung um eine nach §2 Abs. 1a SGBV lebensbedrohliche, regelmäßig tödlich verlaufende oder gleichwertige Erkrankung handelt, denn nur in diesem Fall kann mit einer Kostenerstattung durch die gesetzliche Krankenkasse gerechnet werden. $\mathrm{Zu}$ diesen $\mathrm{Er}$ krankungen gehören die meisten onkologischen Diagnosen, bei vielen Erkrankungen besteht allerdings eine definitorische Unschärfe. Auch werden Erkrankungen mit starkem Verlust der Lebensqualität (z.B. drohende Erblindung) von einzelnen Gerichtsurteilen den lebensbedrohlichen Erkrankungen gleichgestellt.
Klinische Studien

Sollte eine Studie mit dem fraglichen Wirkstoff durchgeführt werden, so sollte eine Prüfung auf Indikation sowie Einund Ausschlusskriterien veranlasst und der Patient bei Einwilligung und Eignung in die Studie eingeschlossen werden. Bei Zulassungsstudien werden die Kosten in der Regel vom Arzneimittel produzierenden Unternehmen getragen.

Tumorkonferenz

Zum interdisziplinären Informationsaustausch aller Hauptbehandlungspartner 
eines Tumorpatienten werden in regelmäßigen Abständen Tumorkonferenzen abgehalten. Chefärzte, niedergelassene Vertragsärzte und weitere an der Behandlung beteiligte Experten besprechen dabei die bestmögliche Therapie des jeweiligen Patienten [7].

Eine Off-Label-Verordnung sollte in dieser Expertenrunde diskutiert werden und auf breite Zustimmung stoßen. Für den Chemotherapie verordnenden Onkologen bedeutet der Informationsaustausch eine zusätzliche Absicherung der Plausibilität der Off-Label-Anwendung.

Die Ergebnisse der Tumorkonferenz sollen dokumentiert werden und bei nicht vorhandener Therapiealternative als Beweis dafür dienen, dass sich eine Expertengruppe mehrheitlich für einen Off-Label-Use ausgesprochen hat und alle Gesichtspunkte dieser Therapie bedacht wurden.

Qualität und Wirksamkeit der Therapie Besonderes Augenmerk soll hier auf die Daten geworfen werden, die von der Expertengruppe Off-Label des BfArM veröffentlicht werden. Nach §35cAbs.1SGBV bilden die Empfehlungen dieser Expertengruppe die Entscheidungsbasis für die gesetzlichen Krankenkassen. Zusätzlich sollte geprüft werden, ob Härtefallprogramme (engl. Compassionate Use, CU) zum fraglichen Arzneistoff vorliegen, in die der Patient eventuell eingeschlossen werden kann. Anders als beim Off-LabelUse werden die Kosten der innerhalb von Härtefall-Programmen angewendeten Medikamente aber vom Arzneimittel produzierenden Unternehmen und nicht von den gesetzlichen Krankenkassen übernommen [8]. Auf der Homepage des BfArM sind alle derzeitigen CU-Programme in Deutschland verzeichnet.

Weitere Aussagen über die Qualität und Wirksamkeit von Wirkstoffen lassen sich vor einer Marktzulassung aus Wirksamkeitsstudien, also Phase-III-Studien, entnehmen. Diese sind laut MDK dann ausreichend, wenn gleichzeitig ein Zulassungsantrag beim BfArM oder der EMA gestellt wurde. Ist kein Zulassungsantrag gestellt, muss zusätzliche Literatur gefunden werden, die den Off-Label-Use rechtfertigt. Hier gilt die Faustregel: Je mehr und je validere Literaturquellen den OffLabel-Einsatz untermauern, desto sicherer ist die Erstattungsfähigkeit. Dabei kann es sich auch um einen Leitlinienkonsens handeln, ohne dass Empfehlun- gen in Leitlinien jedoch allein für die Erstattung des Arzneimittels ausreichend sind, da sie nicht rechtsbindend sind. Bei einer akuten Lebensbedrohung genügen aber Indiz-gestützte Hinweise auf einen möglichen Behandlungserfolg. Hier liegt es zunächst an der - jedoch gerichtlich überprüfbaren - Einschätzung des behandelnden Arztes, ob es sich um eine „akut lebensbedrohliche“ Erkrankung, oder um eine „lebensbedrohliche oder die Lebensqualität auf Dauer nachhaltig beeinträchtigende Erkrankung“ handelt [6].

\section{Dokumentation und Patientenaufklärung} bei notwendiger Off-Label-Anwendung

Zeigt sich am Ende des Algorithmus, dass es weder eine zugelassene Therapiealternative noch eine Studie oder ein Härtefallprogramm gibt, in die der lebensbedrohlich erkrankte Patient eingeschlossen werden kann, so ist der Off-Label-Use als unumgänglich anzusehen. Um dem Regress durch die Krankenkassen vorzugreifen, sollte der verordnende Arzt alle Daten, die zu dieser Off-Label-Anwendung verfügbar sind, sammeln und dokumentieren. Daraufhin ist die gezielte und umfassende Aufklärung des Patienten über den geplanten Off-Label-Use vorzunehmen.

\section{Nutzen und Ziele des Algorithmus zu Off-Label-Verordnungen}

Die Evaluation im Rahmen einer Diplomarbeit ergab, dass der entwickelte Algorithmus in der Lage ist, Off-Label-Verordnungen frühzeitig zu erkennen und zu lenken. In diesem Fall ist in der Regel die Voraussetzung für einen Kostenübernahmeantrag gegeben, der vor Beginn der Therapie bei den Krankenkassen nach $\S 2$ (1a) SGB V eingereicht werden sollte.

Zudem ermöglicht der erarbeitete Algorithmus der Apotheke und dem Medizincontrolling bei Dokumentation der Schritte und unmittelbarer Übermittlung der Daten, sich proaktiv einer Off-LabelVerordnung anzunehmen und diese im besten Falle zu vermeiden oder mit genügend evidenten Daten zu hinterlegen, um eine Erstattung der Therapiekosten durch die Krankenkassen zu erreichen.

Der Sinn des Algorithmus besteht für die therapierende Einrichtung letztlich nicht nur in der Vermeidung von Regressen, sondern - mindestens ebenso bedeutsam - in einer Erhöhung der Therapiesicherheit. Der Arzt wird bei der Verwendung des entwickelten Leitfadens dazu angehalten, seine Therapieplanung kritisch zu überdenken und erhält zusätzliche Vorschläge, wie der Therapieansatz gesichert oder verbessert werden kann. Der Einbezug von aktuellen Studien und nicht zugelassenen Therapiealternativen mit nachgewiesener Evidenz ermöglicht eine Berücksichtigung neuer medizinischer Methoden und führt zu einer möglichst hohen Therapiesicherheit für den Patienten. Diese kann sich unter Umständen auch darin äußern, dass das Ergebnis des Algorithmus eine Empfehlung gegen die Durchführung der geplanten Off-LabelTherapie sein kann.

Die Inhalte dieser Arbeit entstammen einer am Universitätsklinikum Heidelberg erstellten Diplomarbeit mit dem Titel „Off-Label-Use in der Onkologie - Handlungsalgorithmus bei der Verordnung einer Chemotherapie“.

Literatur

1 Späth M. Off-Label-Verordnungen nach dem BSG-Urteil. Hamburger Ärzte Blatt 2002; 9: 393-395

2 Freund VM. On-Label - Off-Label, gibt es eine Sicherheitsgarantie durch Regulation? G+G Wissenschaft 2006; 6: 22-31

3 Cras A, Conscience M-A, Rajzbaum G et al. Off-label prescribing in a French hospital. Pharm World Sci 2007; 29: 97-100. Available from: http://www.ncbi.nlm.nih.gov/pubmed/17160629 (accessed 08 Apr 2013)

4 Radley DC, Finkelstein SN, Stafford RS. Offlabel prescribing among office-based physicians. Arch Intern Med United States 2006; 166: $1021-1026$

5 § 84 Abs.1-3 Gesetz über den Verkehr mit Arzneimitteln, http://www.gesetze-im-internet.de/bundesrecht/amg_1976/gesamt. pdf, Zugriffsdatum 08 Apr 2013

6 Grell L, Rieger M. Off-Label therapy from the perspective of the medical insurance service. Z Rheumatol 2012; 71: 101-107

7 Gerlach K. Lungenkrebszentrum des NCT Heidelberg Thoraxklinik-Heidelberg; Available from: http://www.thoraxklinik-heidelberg.de/index.php?id=42. Accessed $08 \mathrm{Apr}$ 2013

8 Koyuncu, A. Azneimittelversorgung im OffLabel-Use - der rechtliche Rahmen. Deutsche Medizinische Wochenschrift 2012; 137: 1519-1523

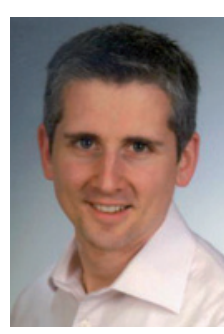

Korrespondenz Dr. Tilman Schöning Universitätsklinikum Heidelberg Apotheke Pharmazeutisch-Onkologischer Service Im Neuenheimer Feld 670 69120 Heidelberg Fax: 06221/56-33424 E-Mail:Tilman.Schoening@ med.uni-heidelberg.de 